\title{
LA MANDRAGORE, NON SEULEMENT UN ANALGESIQUE NARCOTIQUE: SA PLACE DANS LA MEDECINE ET LA CIVILISATION GRECQUES ANTIQUES
}

\author{
Konstantinos LAIOS $^{1 \bowtie}{ }^{\bowtie}$, Grégoire TSOUCALAS ${ }^{2}$, Efstathia LAGIOU ${ }^{1,3}$, \\ Georges ANDROUTSOS ${ }^{4}$, Marianna KARAMANOU ${ }^{1,4}$ \\ ${ }^{1}$ Service d'Histoire de la Médecine et de la Déontologie Médicale, Faculté de Médecine, Université de \\ Crète, Crète, Grèce \\ ${ }^{2}$ Service d'Histoire de la Médecine, Faculté de Médecine, Université Démocrite de Thrace, Alexandroupolis, \\ Grèce \\ ${ }^{3}$ Faculté de Médecine, Université de Patras, Patras, Grèce \\ ${ }^{4}$ Fondation de Recherche Biomédicale de l'Académie d'Athènes, Athènes, Grèce
}

Reçu le 15 Oct 2018, Accepté le 23 Nov 2018

https://doi.org/10.31688/ABMU.2018.53.4.16

\section{Abstract}

The mandrake, not just a narcotic: its place in medicine and ancient greek civilization

Hippocrates and his followers, as well as Pedanius Dioscorides, Pseudo-Galen and Aretaeus of Cappadocia are just a few of the many ancient Greek physicians who have used the plant mandrake. Its narcotic properties were not limited to palliate pain during surgery, but they have been also used to treat pulmonary, neurological and mental diseases. Moreover, mandrake was celebrated in ancient Greek mythology, religion and comedy.

Keywords: Mandrake, ancient Greek medicine, psychiatric and neurological disorders.

\section{Résumé}

Les médecins de la collection Hippocratique, ainsi que Pedanius Dioscorides, Pseudo-Galien et Arétée de Cappadoce sont parmi les médecins Grecs qui ont utilisé la mandragore. Les propriétés narcotiques et analgésiques de cette plante ne se limitent pas à la chirurgie ; la mandragore a été aussi administrée pour le traitement des maladies respiratoires, neurologiques et mentales. En plus, la mandragore a été mentionnée dans la mythologie, la religion et la comédie Grecque antique.

Mots-clés: mandragore, médecine Grecque ancienne, désordres psychiatriques et neurologiques. 


\section{INTRODUCTION}

La mandragore, une plante endémique de Thessalie, est probablement la seule plante qui a beaucoup préoccupé les anciens Grecs. Ses propriétés curatives étaient liées intrinsèquement à la médecine antique Grecque et ses propriétés narcotiques ont donné lieu à développer plusieurs idées mythologiques et religieuses. La mandragore a été utilisée comme médicament dans la chirurgie, mais aussi comme un puissant analgésique. Elle a été également considérée un sédatif dans les cas de maladies pulmonaires ${ }^{1}$. En plus, elle a été administrée avant les interventions chirurgicales en association avec Papaver Somniferum (extrait de pavot-opium) ${ }^{2-3}$. Pour les anciens Grecs, la Nature est celle qui donne la vie et les plantes sont considérées comme des êtres vivants, fixés dans le sol dont la partie supérieure s'épanouit dans l'air ou dans l'eau. Dans la médecine théurgique-magique, la mandragore était réputée pour ces racines, qui ressemblaient à l'homme (Figure 1).

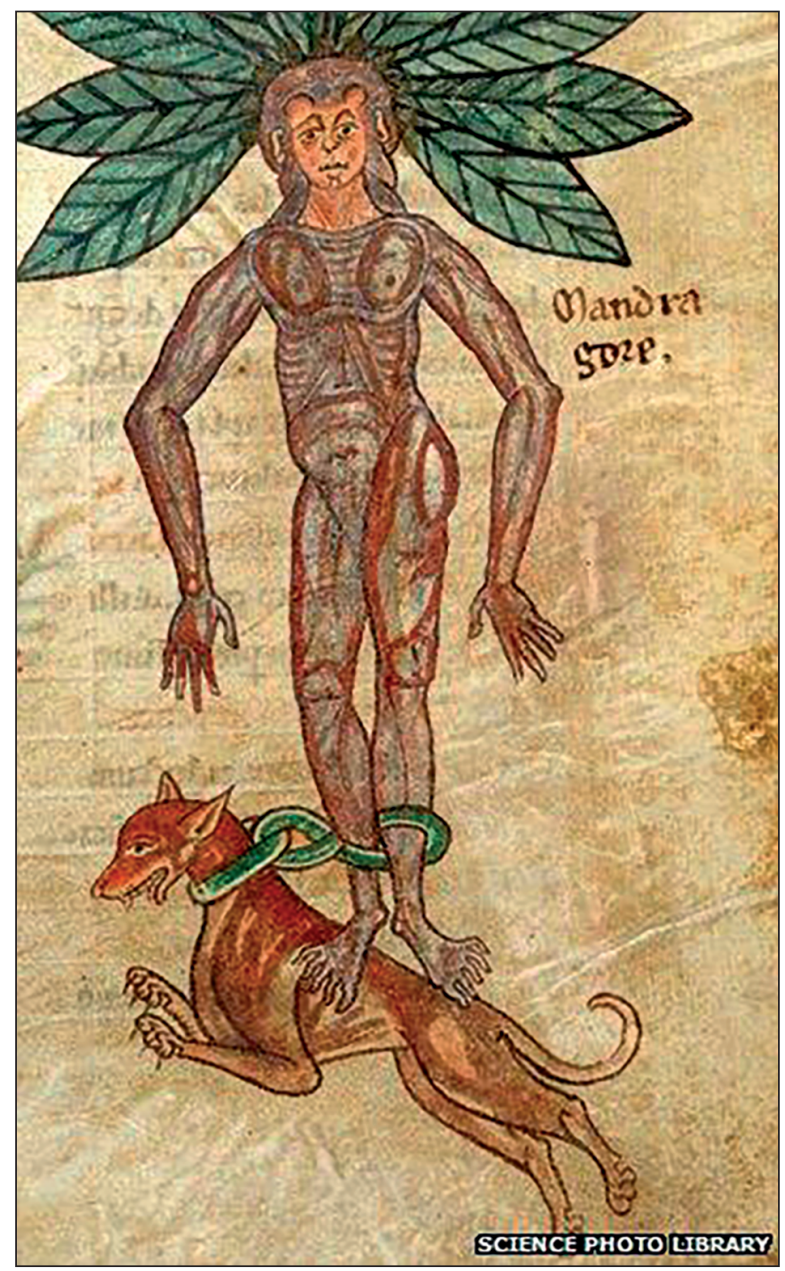

Figure 1. La Mandragore, une illustration publiée aux Pays-Bas vers 1175.
La forme de la racine et le délire causé par la surconsommation, ont donné naissance aux hypothèses sur les vertus et les pouvoirs magiques de la plante ${ }^{4}$.

\section{La mandragore dans la médecine, la mythologie, la religion et la comédie de la Grèce antique}

La mandragore (Mandragora officinarum) (Figure 2) est une plante qui provoque narcose et euphorie. Son action devient le motif afin que les Grecs anciens l'exploitent en modes divers selon leur éducation et leur niveau social. En plus, il était largement admis qu'elle pouvait apporter richesse, fertilité et guérison à celui ou celle qui en possède. Ces idées persistaient au Moyen Âge et même bien après 5 .

Les anciens médecins grecs, toujours raisonnables, ont découvert dans la mandragore un médicament très efficace et utile pour le traitement de plusieurs maladies et le mentionnent souvent dans leurs textes médicaux. Pedanius Dioscoride (c. 40-90 après J.-C.) nous a offert l'analyse la plus détaillée de la mandragore. Il juxtapose aussi ses autres noms Grecs,

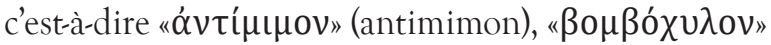

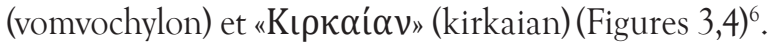
Pseudo-Galien (deuxième siècle après J.-C.) nous informe qu'elle pourrait être remplacée par dorycnium (Dorycnium hirsutum) ${ }^{7}$ et par le coquelicot (Papaver rhoeas $)^{8}$. Les hommes la recevaient sous forme de jus, bouillie, huile et vin mélangé $e^{910}$. Les mêmes résultats

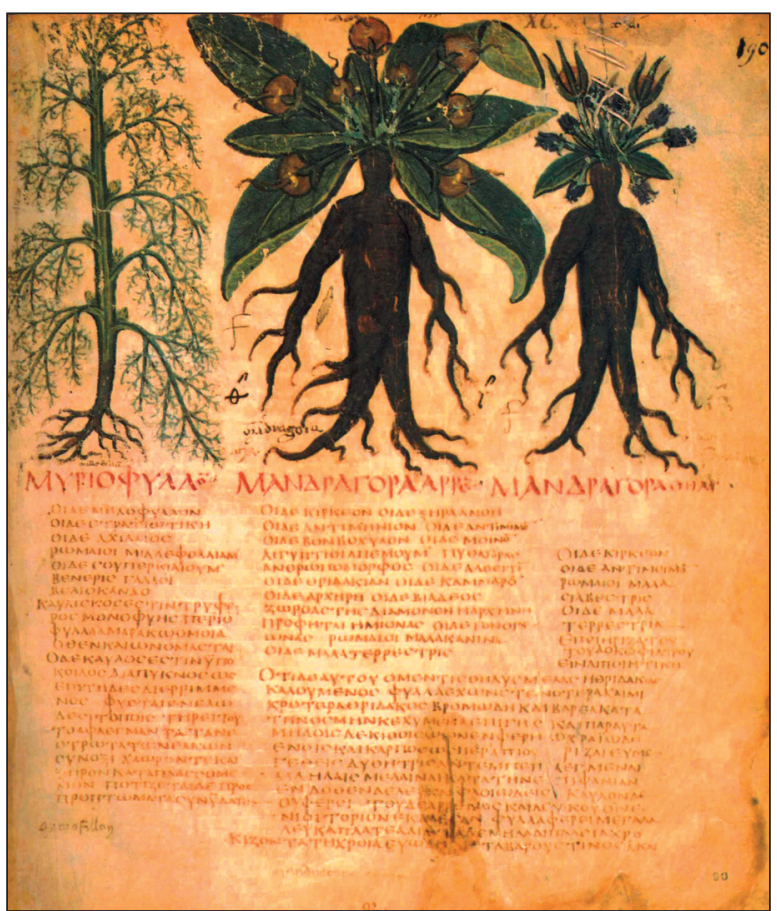

Figure 2. Mandragora officinarum, Herbier général de l'amateur vol. 8, 1817-1827. 


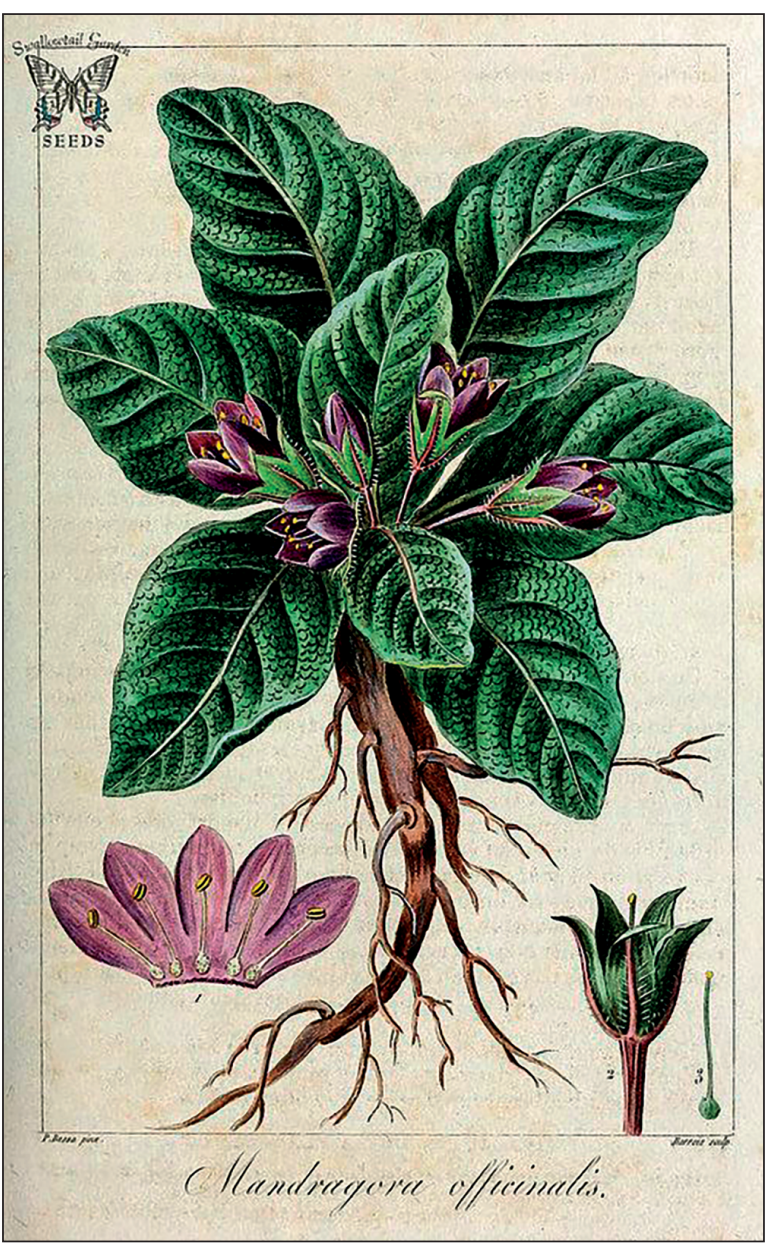

Figure 4. Mandragores mâle et femelle, Manuscrit Dioscurides, Biblioteca Nazionale di Napoli, début du VIIe siècle. étaient présentés, quand on la sentait ou recevait un lavement ${ }^{6}$.

Cependant, la mandragore a été plutôt utilisée comme une potion dormitive, c'est pourquoi la plupart des rapports des médecins anciens Grecs ${ }^{11}$ et des autres écrivains ${ }^{12}$, étaient consacrés à cette action. Aussi essentiel pour les anciens était l'oubli que la mandragore provoquait ${ }^{13}$. Ces deux caractéristiques nous conduisent à la conclusion qu'elle était devenue un médicament pour combattre les désordres psychiatriques et neurologiques A la Collection Hippocratique il y ait eu une recommandation ; administrer la mandragore, quand les patients étaient pris de folie ou voulaient se pendre: "Aux gens tristes, malades et qui veulent s'étrangler, faites prendre le matin en boisson la racine de mandragore à dose moindre qu'il n'en faudrait pour causer le délire " ${ }^{14-16}$. Ses efficacités, narcotiques et analgésiques, l'ont classifiée comme médicament primordial pour l'anesthésie aux opérations et aux cautérisations ${ }^{3,6,17-18}$. En plus, elle était utile comme moyen de réconfort aux maux sévères comme celles d'abdomen ${ }^{19}$, de blessures ${ }^{20}$ et de fistules ${ }^{21}$ et aussi aux problèmes respiratoires, quand il y avait une toux chronique ou intense ${ }^{22}$. Les médecins ont aussi compris qu'elle pourrait devenir un poison mortel en cas de surdosage ${ }^{23}$ et ils soulignaient que les plantes narcotiques provoquaient la froidure au corps humain ${ }^{24}$ qui était augmentée selon la dose.

Il faut aussi mentionner que les anciens médecins Grecs ne favorisaient ou encourageaient jamais l'administration des drogues pour obtenir de

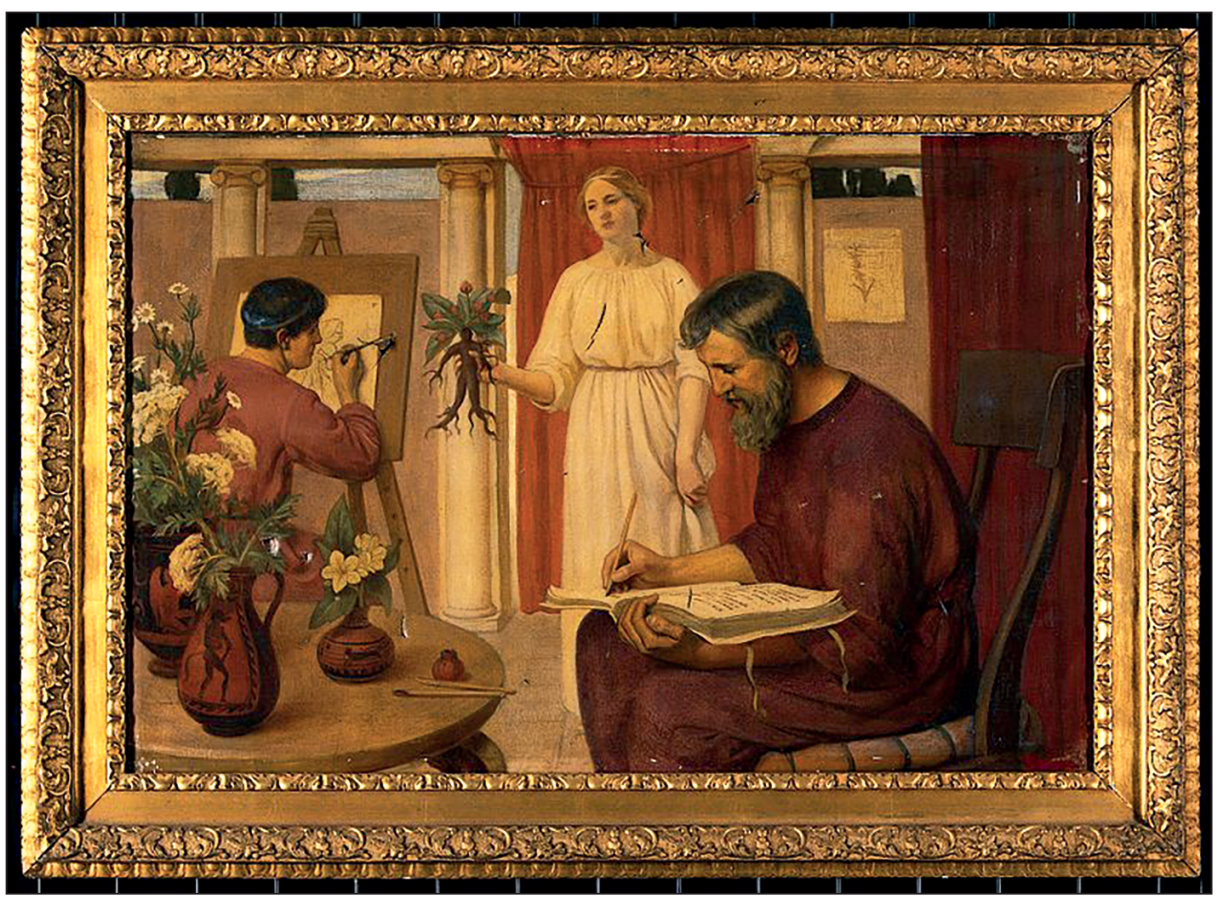

Figure 4. Dioscoride décrivant la mandragore, peinture à l'huile par Ernest Board (1877-1934). 


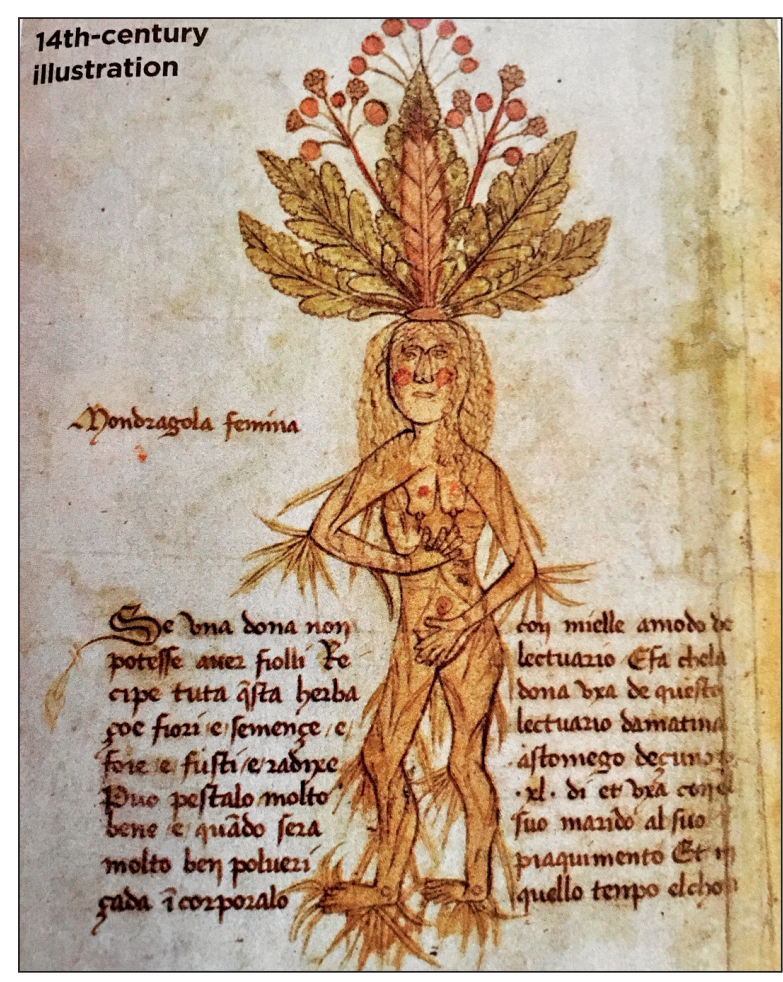

Figure 5. Mandragore (Mandragora), une recette qui aide les femmes à lutter contre l'infertilité et à concevoir des bébés filles. Herbal avec dédicace à Saint Marc, 1591, folio 11, Trento, Biblioteca Del Castello Del Buonconsiglio Monumenti E Collezioni Provinciali.

l'euphorie. Aretaeus de Cappadoce (deuxième ou quatrième siècle après J.-C.), quand il examine la manie, note que cette condition ressemble à la prise de la mandragore ${ }^{25}$. D'autre part, ces caractéristiques étaient l'occasion pour que les hommes sans savoir aient développé des idées religieuses. Théophraste (quatrième - troisième siècle avant J.-C.) nous informe que la mandragore était l'ingrédient principal de la fabrication des filtres magiques ${ }^{26-27}$. Selon ses écrits, il était également utilisé dans des rituels magiques. « tracer autour de la mandragore trois cercles avec une épée, couper en regardant vers le levant, danser autour de l'autre et dire le plus grand nombre possible de paroles grivoises ». Ce rituel s'explique par le fait que, à l'époque de Théophraste les maladies étaient considérées comme une punition divine. Il fallait, dès lors, des remèdes magiques pour détourner l'attention des dieux ${ }^{19}$. Sa qualité comme aphrodisiaque pour les femmes avait une grande réputation, parmi les magiciens et les sorciers, d'éveiller l'amour grâce à ses qualités aphrodisiaques et de guérir la stérilité des femmes (Figure 5) ${ }^{26,28}$.

Dans la mythologie Grecque la plante est corrélée avec la magicienne Circé. Grâce à cette corrélation, elle avait le nom Кıрк $\alpha i ́ \alpha v$ (kirkaian). Le filtre magique de la mandragore a donné à Circé la capacité de transformer les camarades d'Ulysse en animaux et de garder Ulysse ${ }^{29}$. En plus, il existait la conception que la mandragore ait été cultivée au jardin d'Hécate, la déesse du monde des morts, et qu'elle l'a nourrie et guérie. Il y avait aussi les fêtes pour vénérer Zeus et Aphrodite, qui recevaient l'invocation de M $\alpha v \delta \rho \alpha \gamma o \rho \tilde{\imath} \tau \iota \varsigma$ (Mandragoritis) ${ }^{30}$.

La comédie du poète Alexis (quatrième - troisième siècle avant J.-C.) sous le titre "Mandragorisomene " raconte l'histoire d'une femme qui a reçu la mandragore après avoir consulté un médecin. Au fragment qui est conservé, sont décrits les résultats de l'euphorie que la plante provoque ${ }^{31}$.

\section{Conclusion}

Les vertus médicinales de la plante Mandragore étaient connues dans l'antiquité Grecque. La mandragore est un exemple typique du mode de l'utilisation des drogues dans la vie privée et publique des anciens Grecs. Les médecins de l'antiquité ont exploité les propriétés de ce médicament dans diverses maladies et le peuple a créé des idées mythologiques et religieuses.

\section{Aucun conflit d'intérêt}

\section{References}

1. Tsoukalas I. Paediatrics from Homer until nowadays. Science Press, Thessaloniki-Skopelos, 2004.

2. Tsoucalas G, Markatos K, Revelou MT, Androutsos G, Korres D, Karamanou M., Diagoras of Cyprus (3rd century $\mathrm{BC})$ an eminent oculist and oppositor of the use of opium. Arch Balk Med Union 2018;53(3):179-183.

3. Tsoucalas G, Sgantzos M, Androutsos G. Hippocrates, principles on abdominal surgery in ancient Greece during the fifth century B.C. Surg Innov 2016;23(2):212-213.

4. Fabre AJ. Mythologie et plantes médicinales de l'Antiquité. Histoire des Sciences Medicales-Tome XXXVII. 2003;1:65-87.

5. Pauly A, Wissowa G, Kroll W, Witte K, Mittelhaus K, Ziegler K. Mandragoras, Paulys Realencyclopädie der Classischen Altertumswissenschaft, 27, XIV. Stuttgart, JB Metzler, 1894-1980:1028-1038.

6. Dioscorides Pedanius Med. De materia medica 4.75.1.1-3. De materia medica, ed. M. Wellmann, Pedanii Dioscuridis Anazarbei de materia medica libri quinque, 3 vols. Berlin, Weidmann, 1:1907; 2:1906; 3:1914 (repr. 1958): 1:1-255, 2:1-339, 3:1-108.

7. Pseudo-Galenus Med. De succedaneis liber 19.736.1-2. De succedaneis liber, ed. C.G. Kühn, Claudii Galeni opera omnia, vol. 19. Leipzig, Knobloch, 1830 (repr. Hildesheim: Olms, 1965): 721-747.

8. Pseudo-Galenus Med. De succedaneis liber 19.736.8, 19.739.3. De succedaneis liber, ed. C.G. Kühn, Claudii Galeni opera omnia, vol. 19. Leipzig, Knobloch, 1830 (repr. Hildesheim: Olms, 1965): 721-747.

9. Alexander Med. Therapeutica 1.469.6. Therapeutica, ed. T. Puschmann, Alexander von Tralles, vols. 1-2. Vienna, 
Braumüller, 1:1878; 2:1879 (repr. Amsterdam: Hakkert, 1963): 1:441-617; 2:3-585

10. Jouanna J, Villard L. Vin et santé en Grèce ancienne. De Boccard, Rouen, 2002:121, 142.

11. Galenus Med. De temperamentis libri iii 1.585.15. De temperamentis libri iii, ed. G. Helmreich, Galeni de temperamentis libri iii. Leipzig, Teubner, 1904 (repr. Stuttgart: 1969): $1-115$.

12. Stephano H. Thesaurus Graecae Linguae, 9, Graz, Akademische Dr, 1954:560-561.

13. Suidas. Lexicon mu.136.1. Lexicon, ed. A. Adler, Suidae lexicon, 4 vols. [Lexicographi Graeci 1.1-1.4. Leipzig, Teubner, 1.1:1928; 1.2:1931; 1.3:1933; 1.4:1935.

14. Hippocrates Med. et Corp. De locis in homine 39.1-2. De locis in homine, ed. É. Littré, Oeuvres complètes d'Hippocrate, vol. 6. Paris, Baillière, 1849 (repr. Amsterdam: Hakkert, 1962): 276-348.

15. Tsoucalas G, Spengos K, Panayiotakopoulos G, Papaioannou T, Karamanou M. Epilepsy, theories and treatment inside corpus Hippocraticum. Curr Pharm Des 2017;23(42):6369-6372.

16. Laios K, Tsoukalas G, Kontaxaki MI, Karamanou M, Androutsos G. Suicide in ancient Greece. Psychiatriki 2014;25(3):200-2007.

17. Michaud LG. Biographie universelle, ancienne et moderne ou Histoire, par ordre alphabétique, de la vie publique et privée de tous les hommes qui se sont fait remarquer par leurs écrits, leurs actions, leurs talents, leurs vertus ou leurs crimes. Paris, Michaud, 1832:153.

18. Tsoucalas G, Kousoulis AA, Mariolis-Sapsakos T, Sgantzos M. Trepanation Practices in Asclepieia: Systematizing a Neurosurgical Innovation. World Neurosurg 2017;103:501-503.

19. Galenus Med. In Hippocratis de victu acutorum commentaria iv 15.489.9-15.490.2. In Hippocratis de victu acutorum commentaria iv, ed. G. Helmreich, Galeni in Hippocratis de victu acutorum commentaria iv. Corpus medicorum Graecorum, vol. 5.9.1. Leipzig, Teubner, 1914: 117-366.

20. Galenus Med. De simplicium medicamentorum temperamentis ac facultatibus 1 11.404.13-16. De simplicium medicamentorum temperamentis ac facultatibus libri xi, ed. C.G. Kühn, Claudii Galeni opera omnia, vols. 11-12. Leipzig, Knobloch, 1826 (repr. Hildesheim: Olms, 1965): 11:379-892; 12:1-377.
21. Hippocrates Med. et Corp. De fistulis 9.35-7. De fistulis, ed. É. Littré, Oeuvres complètes d'Hippocrate, vol. 6. Paris, Baillière, 1849 (repr. Amsterdam: Hakkert, 1962): 448-460.

22. Galenus Med. De compositione medicamentorum secundum locos libri x 13.70.3-10. De compositione medicamentorum secundum locos libri x, ed. C.G. Kühn, Claudii Galeni opera omnia, vols. 12-13. Leipzig, Knobloch, 12:1826; 13:1827 (repr. Hildesheim: Olms, 1965): 12:378-1007; 13:1-361.

23. Galenus Med. In Hippocratis librum vi epidemiarum commentarii vi 17a.903.11-17a.904.5. In Hippocratis librum vi epidemiarum commentarii vi, ed. E. Wenkebach, Galeni in Hippocratis sextum librum epidemiarum commentaria i-vi. Corpus medicorum Graecorum, vol. 5.10.2.2. Leipzig, Teubner, 1940: 3-351.

24. Galenus Med. De sanitate tuenda libri vi 6.447.4-8. De sanitate tuenda libri vi, ed. K. Koch, Galeni de sanitate tuenda libri vi. Corpus medicorum Graecorum, vol. 5.4.2. Leipzig, Teubner, 1923: 3-198.

25. Aretaeus Med. De causis et signis acutorum morborum 1.6.1.5-6. De causis et signis acutorum morborum (lib. 2), ed. K. Hude, Aretaeus, 2nd edn. Corpus medicorum Graecorum, vol. 2. Berlin, Akademie-Verlag, 1958: 36-90.

26. Theophrastus Phil. Historia plantarum. 9.8.8.1-14, 9.9.1.1-13. Historia plantarum, ed. A. Hort, Theophrastus. Enquiry into plants, 2 vols. Cambridge, Mass, Harvard University Press, 1916 (repr. 1:1968; 2:1961): 1:2-474; 2:2-320.

27. Boardman J, Burkert W, Camporeale G, Graf F, Hermary A, Hölscher T, Lambrinoudakis V, Scheid J, Simon E, Torelli M, True M, et al. Pflazennhebung, Thesaurus Cultus et Rituum Antiquorum, III. Los Angeles, J Paul Getty Museum, 2005: 297.

28. Principaux Archéologues Français et Etrangères. Les Monuments de la Ptérie. Revue Archéologique 1872(23): 282-286.

29. Kirke. Lexicon Iconographicum Mythologiae Classicae, VI, Zürich-München, Verlag,1992: 48-59.

30. Roscher WH. Mandragoras, Ausführliches Lexicon der griechischen und römischen Mythologie, Leipzig, BG Teubner, 1884-1937:2311.

31. Alexis Comic. Fragmenta 141-145.1. Fragmenta, ed. T. Kock, Comicorum Atticorum fragmenta, vol. 2. Leipzig, Teubner, 1884: 297-329, 331-408. 